VOL. $70(2004)$ [229-244]

\title{
FIXED POINT PROPERTY FOR GENERAL TOPOLOGIES IN SOME BANACH SPACES
}

\begin{abstract}
Maria A. Japón Pineda and Stanisław Prus
We study the fixed point property with respect to general vector topologies in $L$ embedded Banach spaces. Considering a class of topologies in $l_{1}$ such that the standard basis is convergent, we characterise those of them for which the fixed point property holds. We show that in $c_{0}$-sums of some Banach spaces the weak topology is in a sense the coarsest topology for which the fixed point property holds.
\end{abstract}

\section{INTRODUCTION}

Every Banach space is equipped with the norm topology and the weak topology. Both of them play important roles in the fixed point theory. In particular, it is possible to characterise sets with the fixed point property for some class of mappings in terms of the weak topology. Such characterisation for the class of continuous affine mappings on bounded convex sets in arbitrary Banach spaces can be found in [3] and for the class of nonexpansive mappings on bounded convex subsets of $c_{0}$ can be found in $[\mathbf{4}, \mathbf{5}]$.

In the case of $L_{1}$ spaces another topology was successfully applied. This is the topology of convergence locally in measure (see [14]). As a generalisation of this topology an abstract measure topology in $L$-embedded Banach spaces was introduced (see [16]). Its applications to the metric fixed point theory can be found in $[11,12]$. In this paper we give another one. Our result concerns existence of fixed points of mappings of asymptotically nonexpansive type in an $L$-embedded Banach space $X$ endowed with a vector topology satisfying the Kadec-Klee property. We study in details the special case when $X=l_{1}$. For a particular family of sets in $l_{1}$ a characterisation of the fixed point property for nonexpansive mappings was found in [8]. Moreover, it is well known that $l_{1}$ has the fixed point property for nonexpansive mappings on convex sets which are compact with respect to the weak* topology generated by the predual $c_{0}$ and lacks this property if we replace $c_{0}$ by $c$. This leads to the problem of characterising locally convex topologies $\tau$ in $l_{1}$ for which the fixed point property holds. We find a solution to this problem in the case when the standard basis of $l_{1}$ is $\tau$-convergent.

In the last section of this paper we deal with $c_{0}$-sums of reflexive spaces. Using an idea from [4] we show that for some such spaces the weak topology is in a sense the coarsest topology for which the fixed point property holds.

Received 15th March, 2004

Copyright Clearance Centre, Inc. Serial-fee code: 0004-9727/04 \$A2.00+0.00. 


\section{Preliminaries}

Let $X$ be a normed space. Its closed unit ball will be denoted by $B_{X}$. By a vector topology in $X$ we mean a Hausdorff topology $\tau$ such that the vector operations are continuous with respect to $\tau$. Given a subspace $Y$ of the dual space $X^{*}$, by $\sigma(X, Y)$ we denote the coarsest topology in $X$ for which all functionals $f \in Y$ are continuous. Recall that $Y$ is total if for every $x \in X \backslash\{0\}$ there exists $f \in Y$ such that $f(x) \neq 0$. In this case $\sigma(X, Y)$ is a vector topology. Of course $X^{*}$ is total and $\sigma\left(X, X^{*}\right)$ is just the weak topology which we denote also by $w$. If $X=Y^{*}$, then $Y$ considered as a subspace of $X^{*}$ is total and $\sigma(X, Y)$ is the weak ${ }^{*}$ topology. Another examples can be obtained for Banach spaces $X$ which are not reflexive. Then $\operatorname{ker} F$ is total for every $F \in X^{* *} \backslash X$.

We shall also deal with the so-called abstract measure topologies. Let us recall that a sequence $\left(x_{n}\right)$ in a Banach space $X$ spans an asymptotically isometric copy of $l_{1}$ if there exists a nonincreasing sequence $\left(\delta_{n}\right)$ in $[0,1)$ tending to 0 such that

$$
\sum_{n=1}^{\infty}\left(1-\delta_{n}\right)\left|\alpha_{n}\right| \leqslant\left\|\sum_{n=1}^{\infty} \alpha_{n} x_{n}\right\| \leqslant \sum_{n=1}^{\infty}\left|\alpha_{n}\right|
$$

for every sequence $\left(\alpha_{n}\right) \in l_{1}$. In this case we write $\left(x_{n}\right) \sim\left(\right.$ asy) $l_{1}$. We say that a topology $\tau$ in a Banach space $X$ is an abstract measure topology provided that a norm bounded sequence $\left(x_{n}\right)$ in $X$ converges to $x$ with respect to $\tau$ if and only if every subsequence $\left(y_{n}\right)$ of $\left(x_{n}-x\right)$ has a subsequence $\left(y_{n_{k}}\right)$ such that either $\left(y_{n_{k}} /\left\|y_{n_{k}}\right\|\right) \sim($ asy $) l_{1}$ or $\lim _{k \rightarrow \infty}\left\|y_{n_{k}}\right\|$ $=0$.

Some vector topologies are abstract measure topologies. Let $X=L_{1}(\Omega, \mu)$ where $\mu$ is a $\sigma$-finite measure on a $\sigma$-field of subsets of $\Omega$. Then the topology of convergence locally in measure is an abstract measure topology (see [16]). In the particular case when $X=l_{1}$ this topology coincides with the topology of coordinatewise convergence. On $B_{X}$ this is just the weak* topology $\sigma\left(l_{1}, c_{0}\right)$. The Bergman space $A^{1}$ provides another such example. To recall the definition of $A^{1}$ we put $\mathbb{D}=\{z \in \mathbb{C}:|z|<1\}$ and consider the normalised Lebesgue measure $\mu$ on $\mathbb{D}$. $A^{1}$ is the subspace of $L_{1}(\mathbb{D}, \mu)$ consisting of all analytic functions on $\mathbb{D}$. It is a dual space and for bounded sequences weak ${ }^{*}$ convergence is equivalent to uniform convergence on compact sets (see [15]). This shows that the weak* topology is finer than the topology of convergence in measure on $B_{A^{1}}$ and consequently, these two topologies coincide on $B_{A^{1}}$. The weak ${ }^{*}$ topology in $A^{1}$ is therefore an abstract measure topology.

Let $\tau$ be a vector topology in a Banach space $X$. A function $f: X \rightarrow \mathbb{R}$ is sequentially lower semicontinuous with respect to $\tau$ if

$$
f(x) \leqslant \liminf _{n \rightarrow \infty} f\left(x_{n}\right)
$$

for every sequence $\left(x_{n}\right)$ in $X$ which converges to $x$ with respect to $\tau$. Observe that 'lim inf' may be replaced by 'limsup' in this definition. The space $X$ has the Kadec-Klee property 
with respect to $\tau$ provided that if $\left(x_{n}\right)$ is a sequence in $X$ without a norm convergent subsequence and $\left(x_{n}\right)$ converges to $x$ with respect to $\tau$, then

$$
\|x\|<\limsup _{n \rightarrow \infty}\left\|x_{n}\right\| .
$$

If $\tau$ is coarser than the norm topology, then the Kadec-Klee $(\tau)$ property implies that the norm $\|\cdot\|$ is $\tau$-sequentially lower semicontinuous.

Let $C$ be a nonempty subset of $X$. A mapping $T: C \rightarrow C$ is nonexpansive if

$$
\|T(x)-T(y)\| \leqslant\|x-y\|
$$

for all $x, y \in C$. In the case when strict inequality holds in the above condition whenever $x \neq y$, we say that $T$ is contractive. A mapping $T: C \rightarrow C$ is of asymptotically nonexpansive type if $T^{N}$ is continuous for some $N \in \mathbb{N}$ and

$$
\limsup _{n \rightarrow \infty}\left(\sup \left\{\left\|T^{n}(x)-T^{n}(y)\right\|-\|x-y\|: y \in C\right\}\right) \leqslant 0
$$

for every $x \in C$. The space $X$ has the $\tau$-fixed point property provided that if $C$ is a nonempty bounded convex and $\tau$-sequentially compact subset of $X$ and $T: C \rightarrow C$ is nonexpansive, then $T$ has a fixed point. A mapping $T: C \rightarrow C$ is said to satisfy the $(P)_{T}$-fixed point property if $T$ has a fixed point in every nonempty convex $\tau$-sequentially closed subset $D$ of $C$ such that if $x \in D$, then each $\tau$-limit of a subsequence of $\left(T^{n}(x)\right)$ belongs to $D$.

Let $\tau$ be a vector topology in a space $X$. In the sequel $\tau_{B_{X}}$ will denote the restriction of the topology $\tau$ to the ball $B_{X}$. We say that $\tau$ is coarser than the weak topology on the unit ball if $\tau_{B_{X}}$ is coarser than $w_{B_{X}}$.

We shall consider mainly locally convex topologies, that is, vector topologies which admit local bases consisting of convex sets. Let $\tau$ be such a topology in a space $X$ and $E$ be the space dual to $(X, \tau)$. Given nonempty sets $A \subset X, D \subset E$, we consider the polar sets

$$
A^{\circ}=\left\{f \in E: \sup _{x \in A}|f(x)| \leqslant 1\right\}
$$

and

$$
D_{\circ}=\left\{x \in X: \sup _{f \in D}|f(x)| \leqslant 1\right\} .
$$

Proposition 1. Let $X$ be a normed space and $Y=(X, \tau)$ where $\tau$ is a locally convex topology in $X$ coarser than the weak topology on the unit ball. Then a bounded sequence $\left(x_{n}\right)$ converges to $x$ with respect to the topology $\sigma\left(X, Y^{*}\right)$ if and only if $\left(x_{n}\right)$ converges to $x$ with respect to $\tau$.

Proof: Our assumption guarantees that $\tau$ is coarser than the norm topology. This shows in particular that $Y^{*} \subset X^{*}$. Let $U$ be a convex, balanced and $\tau$-closed neighbourhood of zero. There is $r>0$ such that $r B_{X} \subset U$. It follows that the polar $U^{\circ}$ of $U$ in $Y^{*}$ is bounded. 
By our assumption for each $\varepsilon>0$ there is a finite set $F \subset X^{*}$ such that $F_{\circ} \cap B_{X} \subset \varepsilon U$. We put $Z=\bigcap_{x^{*} \in F} \operatorname{ker} x^{*}$. Then $Z \subset F_{\circ}$ which yields

$$
\frac{1}{\varepsilon} U^{\circ}=(\varepsilon U)^{\circ} \subset\left(Z \cap B_{X}\right)^{\circ}=\left(B_{Z}\right)^{\circ}
$$

Moreover, if $g \in\left(B_{Z}\right)^{\circ}$, then we can find $y^{*} \in X^{*}$ so that $\left\|y^{*}\right\|=\left\|g_{\mid Z}\right\| \leqslant 1$ and $y_{\mid Z}^{*}=g_{\mid Z}$. Thus $h_{\mid Z}=0$ where $h=g-y^{*}$. It follows that $h \in \operatorname{span}(F)$. We therefore see that $\left(B_{Z}\right)^{\circ} \subset \operatorname{span}(F)+B_{X^{*}}$. Hence

$$
U^{\circ} \subset \operatorname{span}(F)+\varepsilon B_{X^{*}}
$$

Using this fact, one can easily show that the set $U^{\circ}$ is relatively compact in the norm topology.

Let now $\left(x_{n}\right)$ be a bounded sequence in $X$ converging to $x$ with respect to $\sigma\left(X, Y^{*}\right)$. We can assume that $x=0$ and $\left(x_{n}\right)$ is contained in $B_{X}$. For every convex balanced $\tau$ closed neighbourhood $U$ of zero we find a finite $1 / 2$-net $\left\{f_{1}, \ldots, f_{m}\right\}$ in $U^{\circ}$. There exists $n_{0} \in \mathbb{N}$ such that $\max _{1 \leqslant k \leqslant m}\left|f_{k}\left(x_{n}\right)\right| \leqslant 1 / 2$ for every $n \geqslant n_{0}$. Given $f \in U^{\circ}$, we choose $k$ for which $\left\|f-f_{k}\right\| \leqslant 1 / 2$. Then

$$
\left|f\left(x_{n}\right)\right| \leqslant\left|f_{k}\left(x_{n}\right)\right|+\left\|f-f_{k}\right\| \leqslant 1
$$

which shows that $x_{n} \in\left(U^{\circ}\right)_{0}$ for every $n \geqslant n_{0}$. But by the bipolar theorem (see [13]), $\left(U^{\circ}\right)_{0}=U$. We therefore see that $\left(x_{n}\right)$ converges to $x$ with respect to $\tau$. The remaining part of the conclusion is obvious.

Corollary 2. Let $X$ be a normed space and $Y=(X, \tau)$ where $\tau$ is a locally convex topology in $X$ coarser than the weak topology on the unit ball. If $\left(x_{n}\right)$ is a bounded sequence in $X$ converging to $x$ with respect to $\sigma\left(X, Y^{*}\right)$, then the set

$$
C=\left\{t_{0} x+\sum_{n=1}^{\infty} t_{n} x_{n}: \sum_{n=0}^{\infty} t_{n}=1, t_{n} \geqslant 0, n=0,1,2, \ldots\right\}
$$

is $\tau$-sequentially compact.

Proof: Consider the mapping $\Phi: l_{1} \rightarrow X$ given by the formula

$$
\Phi\left(\lambda_{1}, \lambda_{2}, \ldots\right)=\lambda_{1} x+\sum_{j=2}^{\infty} \lambda_{j} x_{j}
$$

Using Proposition 1, one can easily show that $\Phi$ is $\sigma\left(l_{1}, c\right)$ to $\tau$ sequentially continuous. It suffices now to observe that $C=\Phi(K)$ where

$$
K=\left\{\left(\lambda_{1}, \lambda_{2}, \ldots\right) \in l_{1}: \sum_{n=1}^{\infty} \lambda_{n}=1, \lambda_{n} \geqslant 0, n=1,2, \ldots\right\}
$$


is sequentially compact with respect to $\sigma\left(l_{1}, c\right)$.

Let $\tau$ be a locally convex topology in a space $X$. A modification of the reasoning used in the proof of Proposition 1 shows that if a convex balanced $\tau$-closed neighbourhood $U$ of zero contains an open weak neighbourhood of zero, then $\operatorname{span}\left(U^{\circ}\right)$ is a finite dimensional subspace of $Y^{*}$. Since $U^{\circ}$ is bounded, it is contained in an absolute convex hull of a finite set $A \subset Y^{*}$. Consequently, $A_{\circ} \subset\left(U^{\circ}\right)_{\circ}=U$. This shows that if a locally convex topology $\tau$ in $X$ is coarser than the weak topology, then $\tau=\sigma\left(X, Y^{*}\right)$.

The assumption of Proposition 1 does not guarantee this conclusion. Indeed, let $X$ be an infinite dimensional normed space and $\mathcal{B}$ be the family of all polar sets $A_{\circ}$ where $A$ is a nonempty compact subset of $X^{*}$. Then $\mathcal{B}$ is a local basis at zero of a locally convex topology $\tau$ in $X$ which is finer than the weak topology. Consequently, $X^{*}$ is the dual space of $(X, \tau)$. If $A \subset X^{*}$ is a compact set which is not contained in any finite dimensional subspace of $X^{*}$, then $A_{\circ}$ does not contain any open weak neighbourhood of zero. This shows that $\tau$ does not coincide with the weak topology. On the other hand, it is easy to see that $\tau_{B_{X}}$ coincides with $w_{B_{X}}$.

\section{L-EMBEDDED SPACES}

Let us recall that $X$ is an $L$-embedded Banach space if there exists a closed subspace $Z$ of $X^{* *}$ such that $X^{* *}=X \oplus Z$ and $\|x+z\|=\|x\|+\|z\|$ for all $x \in X$ and $z \in Z$. In particular every space $L_{1}(\Omega, \mu)$ is an $L$-embedded space. For thorough study of $L$ embedded spaces the reader may consult the monograph [9]. In [12], the following property of $L$-embedded spaces was established.

Proposition 3. Let $X$ be an L-embedded Banach space. If a bounded sequence $\left(x_{n}\right)$ converges to 0 in an abstract measure topology, then

$$
\limsup _{n \rightarrow \infty}\left\|x+x_{n}\right\|=\|x\|+\limsup _{n \rightarrow \infty}\left\|x_{n}\right\|
$$

for every $x \in X$.

Proposition 3 will be used many times in this paper. As the first application we obtain the following lemma.

Lemma 4. Let $\tau$ be a vector topology in an L-embedded Banach $X,\left(x_{n}\right)$ be a bounded sequence in $X$ such that the set $\left\{x_{n}\right\}$ is relatively sequentially compact in an abstract measure topology and

$$
r(x)=\limsup _{n \rightarrow \infty}\left\|x_{n}-x\right\|
$$

where $x \in X$.

(i) If the norm of $X$ is $\tau$-sequentially lower semicontinuous, then the function $r$ is $\tau$-sequentially lower semicontinuous. 
(ii) If $X$ has the Kadec-Klee $(\tau)$ property and $\left(z_{n}\right)$ is a sequence in $X$ such that $\left(z_{n}\right)$ converges to $z$ with respect to $\tau$ and $\left(z_{n}\right)$ does not have a norm convergent subsequence, then

$$
r(z)<\limsup _{n \rightarrow \infty} r\left(z_{n}\right)
$$

ProOF: Let a sequence $\left(z_{n}\right)$ converge to $z$ with respect to $\tau$. We find a sequence $\left(n_{k}\right)$ so that $r(z)=\lim _{k \rightarrow \infty}\left\|x_{n_{k}}-z\right\|$ and $\left(x_{n_{k}}\right)$ converges to some $y$ with respect to the abstract measure topology. Then using Proposition 3, we obtain

$$
\begin{aligned}
r(z) & =\|y-z\|+\limsup _{k \rightarrow \infty}\left\|x_{n_{k}}-y\right\| \leqslant \underset{m \rightarrow \infty}{\limsup }\left\|y-z_{m}\right\|+\limsup _{k \rightarrow \infty}\left\|x_{n_{k}}-y\right\| \\
& =\limsup _{m \rightarrow \infty} \limsup _{k \rightarrow \infty}\left\|x_{n_{k}}-z_{m}\right\| \leqslant \limsup _{m \rightarrow \infty} r\left(z_{m}\right) .
\end{aligned}
$$

This completes the proof of (i) and the proof of (ii) is similar.

We can now prove our general fixed point results for $L$-embedded spaces.

THEOREM 5. Let $X$ be an $L$-embedded Banach space and $\tau$ be a vector topology in $X$ coarser than the norm topology such that every $\tau$-sequentially compact subset of $X$ is $\tau$-compact and $X$ has the Kadec-Klee $(\tau)$ property. Let a nonempty bounded convex set $C \subset X$ be $\tau$-sequentially compact and relatively sequentially compact in an abstract measure topology. Then every mapping $T: C \rightarrow C$ of asymptotically nonexpansive type has the $(P)_{\tau}$-fixed point property.

Proof: We follow a reasoning form [18]. Let $T: C \rightarrow C$ be a mapping of asymptotically nonexpansive type. We put

$$
r_{x}(y)=\underset{n \rightarrow \infty}{\limsup }\left\|T^{n}(x)-y\right\|
$$

where $x, y \in C$. Clearly,

$$
\begin{aligned}
\left\|T^{n}(x)-T^{m}(y)\right\| & =\left\|T^{m}(y)-T^{m}\left(T^{n-m}(x)\right)\right\|-\left\|y-T^{n-m}(x)\right\|+\left\|y-T^{n-m}(x)\right\| \\
& \leqslant \sup \left\{\left\|T^{m}(y)-T^{m}(v)\right\|-\|y-v\|: v \in C\right\}+\left\|T^{n-m}(x)-y\right\|
\end{aligned}
$$

for all $x, y \in C$ and $n>m$. Hence

$$
\limsup _{m \rightarrow \infty} \limsup _{n \rightarrow \infty}\left\|T^{n}(x)-T^{m}(y)\right\| \leqslant r_{x}(y) .
$$

Let $\mathcal{F}$ be the family of all nonempty convex $\tau$-sequentially closed subsets $K$ of $C$ such that if $y \in K$ and $z$ is a limit with respect to $\tau$ of a subsequence of $\left(T^{n}(y)\right)$, then $z \in K$. We fix $D \in \mathcal{F}$. From the Zorn lemma it follows that there exists $K_{0} \in \mathcal{F}$ which is minimal with respect to inclusion in the family $\{K \in \mathcal{F}: K \subset D\}$. Let $x \in K_{0}$. We 
shall show that the set $\left\{T^{n}(x)\right\}$ is relatively compact in the norm topology. Let $K_{1}$ be the set of all $z \in K_{0}$ at which the function $r_{x}$ attains its infimum on $K_{0}$. Lemma 4 shows that $K_{1}$ is nonempty and $\tau$-sequentially closed. Obviously it is also convex. Let $z \in K_{1}$ and $\left(n_{k}\right)$ be an increasing sequence such that $\left(T^{n_{k}}(z)\right)$ converges to some $u$ with respect to $\tau$. By Lemma 4 and (1)

$$
r_{x}(u) \leqslant \limsup _{k \rightarrow \infty} r_{x}\left(T^{n_{k}}(z)\right) \leqslant r_{x}(z) .
$$

This shows that $u \in K_{1}$ and we see that $K_{1} \in \mathcal{F}$. Consequently, $K_{1}=K_{0}$ and in particular $r_{x}$ attains at $x$ its infimum on $K_{0}$.

Suppose that there exists an increasing sequence $\left(n_{k}\right)$ such that $\left(T^{n_{k}}(x)\right)$ does not have a norm convergent subsequence. We can assume that $\left(T^{n_{k}}(x)\right)$ converges to some $u \in K_{0}$ with respect to $\tau$. Then Lemma 4 and (1) show that

$$
r_{x}(u)<\limsup _{k \rightarrow \infty} r_{x}\left(T^{n_{k}}(x)\right) \leqslant r_{x}(x)
$$

which is a contradiction. Now it suffices to use the reasoning from the proof of $[18$, Lemma 2].

If the norm of $X$ is not only $\tau$-sequentially lower semicontinuous, but $\tau$-lower semicontinuous, then the assumptions of Theorem 5 actually guarantee that there exists a nonexpansive retraction $R$ from $C$ onto the set $\operatorname{Fix}(T)$ of all fixed points of $T$ such that $R \circ T=R$ and every convex $\tau$-sequentially closed $T$-invariant subset of $C$ is also $R$-invariant (see $[17,1]$ where only the case of $\tau=w$ is considered).

The formulation of Theorem 5 can be simplified if the space $X$ admits an abstract measure topology such that bounded sets are relatively sequentially compact. Further simplification is possible if $X$ is separable. Then $\tau$-sequentially compact sets are $\tau$ compact (see [11]). Both remarks apply for instance to the spaces $A^{1}$ and $l_{1}$.

COROLLARY 6 . Let $\tau$ be a vector topology in $l_{1}$ coarser than the norm topology such that $l_{1}$ has the Kadec-Klee $(\tau)$ property. If a nonempty bounded convex set $C \subset X$ is $\tau$-sequentially compact, then every mapping $T: C \rightarrow C$ of asymptotically nonexpansive type has the $(P)_{\tau}$-fixed point property. In particular $l_{1}$ has the $\tau$-fixed point property.

In Corollary 6 we obtained a condition sufficient for the $\tau$-fixed point property in $l_{1}$. Our next result gives a necessary condition. Before passing to this theorem we establish some notation. Let $\Gamma$ be a nonempty set. Given $x \in l_{1}(\Gamma)$, we write $x=(x(i))_{i \in \Gamma}$ where $x(i)$ are scalars. If $x \neq 0$, we set supp $x=\{i \in \Gamma: x(i) \neq 0\}$. Even if $\Gamma$ is uncountable, this set is at most countable.

THEOREM 7. Let $\Gamma$ be an infinite set and $\tau$ be a locally convex topology in $l_{1}(\Gamma)$ coarser than the weak topology on the unit ball. If the standard norm of $l_{1}(\Gamma)$ is not $\tau$-sequentially lower semicontinuous, then there exist a bounded convex $\tau$-sequentially 
compact set $C \subset l_{1}(\Gamma)$ and a contractive mapping $T: C \rightarrow C$ which does not have a fixed point.

PROOF: By the assumption there exists a sequence $\left(x_{n}\right)$ in $l_{1}(\Gamma)$ such that $\left(x_{n}\right)$ converges to $x$ with respect to $\tau$ and

$$
\|x\|>\lim _{n \rightarrow \infty}\left\|x_{n}\right\|
$$

We can assume that $\left(x_{n}\right)$ converges coordinatewise to some $y$. Setting $u_{n}=x_{n}-y$ and $u_{0}=x-y$, we obtain a sequence $\left(u_{n}\right)$ which converges to $u_{0}$ with respect to $\tau$ and converges coordinatewise to 0 . Then $\left(u_{n}\right)$ does not converge to 0 in norm and by Proposition 3

$$
\left\|u_{0}\right\| \geqslant\|x\|-\|y\|>\lim _{n \rightarrow \infty}\left\|x_{n}\right\|-\|y\|=\lim _{n \rightarrow \infty}\left\|u_{n}\right\| .
$$

We can assume that $A_{n}=\operatorname{supp} u_{n}$ is finite, $\left\|u_{n}\right\|=1$ for every $n \geqslant 1$ and the sets $A_{n}$ are pairwise disjoint. Then $\left\|u_{0}\right\|>1+\varepsilon$ for some $\varepsilon>0$ and there are vectors $u_{0}^{\prime}, u_{0}^{\prime \prime}$ such that $u_{0}=u_{0}^{\prime}+u_{0}^{\prime \prime},\left\|u_{0}^{\prime}\right\|-\left\|u_{0}^{\prime \prime}\right\|>1+\varepsilon, A=\operatorname{supp} u_{0}^{\prime}$ is finite and $A \cap \operatorname{supp} u_{0}^{\prime \prime}=\emptyset$. We can also assume that $A \cap A_{n}=\emptyset$ for every $n \geqslant 1$.

We put $v_{k}=(1+\varepsilon / k) u_{k}$ for $k \geqslant 1$ and

$$
C=\left\{\lambda_{0} u_{0}+\sum_{j=1}^{\infty} \lambda_{j} v_{j}: \sum_{j=0}^{\infty} \lambda_{j}=1, \lambda_{j} \geqslant 0, j=0,1,2, \ldots\right\} .
$$

Clearly, $C$ is bounded and convex. Corollary 2 shows that $C$ is $\tau$-sequentially compact. We now set

$$
T\left(\lambda_{0} u_{0}+\sum_{j=1}^{\infty} \lambda_{j} v_{j}\right)=\sum_{j=0}^{\infty} \lambda_{j} v_{j+1}
$$

This formula defines a mapping $T: C \rightarrow C$ without a fixed point. Moreover,

$$
\begin{aligned}
\left\|\sum_{j=0}^{\infty} \gamma_{j} v_{j+1}\right\| & =\sum_{j=0}^{\infty}\left|\gamma_{j}\right|\left(1+\frac{\varepsilon}{j+1}\right)<\left|\gamma_{0}\right|\left(\left\|u_{0}^{\prime}\right\|-\left\|u_{0}^{\prime \prime}\right\|\right)+\left\|\sum_{j=1}^{\infty} \gamma_{j} v_{j}\right\| \\
& =\left\|\gamma_{0} u_{0}^{\prime}+\sum_{j=1}^{\infty} \gamma_{j} v_{j}\right\|-\left\|\gamma_{0} u_{0}^{\prime \prime}\right\| \leqslant\left\|\gamma_{0} u_{0}+\sum_{j=1}^{\infty} \gamma_{j} v_{j}\right\|
\end{aligned}
$$

for every nonzero $\left(\gamma_{n}\right) \in l_{1}$, which shows that $T$ is contractive.

Theorem 7 may be extended to spaces of the form $\left(\sum_{i \in \Gamma} X_{i}\right)_{l_{1}(\Gamma)}$ where $X_{i}$ are finite dimensional.

Assume that $\tau$ is a vector topology in $l_{1}$ such that if $\left(y_{n}\right)$ converges to $y$ with respect to $\tau$ and converges to 0 coordinatewise, then

$$
\|y\| \leqslant \limsup _{n \rightarrow \infty}\left\|y_{n}\right\|
$$


Then the norm $\|\cdot\|$ is $\tau$-sequentially lower semicontinuous. Indeed, let a bounded sequence $\left(x_{n}\right)$ converge to $x$ with respect to $\tau$. Passing to a subsequence, we can assume that $\left(x_{n}\right)$ converges coordinatewise to some $z$. By our assumption and Proposition 3

$$
\|x\| \leqslant\|x-z\|+\|z\| \leqslant \underset{n \rightarrow \infty}{\limsup }\left\|x_{n}-z\right\|+\|z\|=\limsup _{n \rightarrow \infty}\left\|x_{n}\right\| .
$$

A similar remark applies to the Kadec-Klee $(\tau)$ property.

Under an additional assumption we can give a simple characterisation of topologies $\tau$ for which $l_{1}$ has the $\tau$-fixed point property. By $\left(e_{n}\right)$ we denote the standard basis of $l_{1}$.

THEOREM 8 . Let $\tau$ be a locally convex topology in the real space $l_{1}$ coarser than the weak topology on the unit ball. Assume that $\left(e_{n}\right)$ converges to some $e \in l_{1}$ with respect to $\tau$. Then $l_{1}$ has the $\tau$-fixed point property if and only if one of the following conditions holds

(i) $\|e\|<1$

(ii) $\|e\|=1$ and the set $N^{+}=\{n \in \mathbb{N}: e(n) \geqslant 0\}$ is finite.

Proof: Given $z=(z(k))_{k \in \mathbb{N}} \in l_{1}$ we set

$$
s(z)=\sum_{k=1}^{\infty} z(k)
$$

Moreover, we put

$$
P_{n}(z)=\sum_{k=1}^{n} z(k) e_{k}
$$

where $n \in \mathbb{N}$.

Consider a bounded sequence $\left(x_{n}\right)$ in $l_{1}$ which converges to $x$ with respect to $\tau$ and converges to 0 coordinatewise. If additionally the limit $s=\lim _{n \rightarrow \infty} s\left(x_{n}\right)$ exists, then $x=s e$. Indeed, let $Y$ be the space dual to $\left(l_{1}, \tau\right)$. If $x^{*} \in Y$, then

$$
\begin{aligned}
\left|x^{*}\left(x_{n}\right)-s\left(x_{n}\right) x^{*}(e)\right| & =\left|\sum_{k=1}^{\infty} x_{n}(k)\left(x^{*}\left(e_{k}\right)-x^{*}(e)\right)\right| \\
& \leqslant\left\|x^{*}\right\|(1+\|e\|)\left\|P_{m}\left(x_{n}\right)\right\|+\sup _{k>m}\left|x^{*}\left(e_{k}\right)-x^{*}(e)\right|\left\|x_{n}\right\|
\end{aligned}
$$

for every $m$. It follows that

$$
\left|x^{*}(x-s e)\right|=\lim _{n \rightarrow \infty}\left|x^{*}\left(x_{n}\right)-s\left(x_{n}\right) x^{*}(e)\right| \leqslant \lim _{m \rightarrow \infty} \sup _{k>m}\left|x^{*}\left(e_{k}\right)-x^{*}(e)\right| \limsup _{n \rightarrow \infty}\left\|x_{n}\right\|=0 .
$$

The subspace $Y$ is total, so we obtain the desired formula $x=s e$.

Assume now that (i) or (ii) holds. Then $\|e\| \leqslant 1$. Let $\left(x_{n}\right)$ be a bounded sequence in $l_{1}$ which converges to $x$ with respect to $\tau$ and converges to 0 coordinatewise. Passing to a subsequence, we can assume that the limit $s=\lim _{n \rightarrow \infty} s\left(x_{n}\right)$ exists. Then

$$
\|x\| \leqslant|s|=\lim _{n \rightarrow \infty}\left|s\left(x_{n}\right)\right| \leqslant \limsup _{n \rightarrow \infty}\left\|x_{n}\right\|
$$


This shows that the norm of $l_{1}$ is $\tau$-sequentially lower semicontinuous.

Assume that $l_{1}$ does not have the $\tau$-fixed point property. Lemma 4 enables us to use a generalised Goebel-Karlovitz lemma (see [2, Lemma 1] and [10, Lemma 2.6]) and obtain a sequence $\left(x_{n}\right)$ such that it converges to $x_{0}$ with respect to $\tau$ and $\lim _{n \rightarrow \infty}\left\|u-x_{n}\right\|=2$ for every $u \in \operatorname{conv}\left\{x_{n}: n \geqslant 0\right\}$. We can assume that $\left(x_{n}\right)$ converges coordinatewise to some $y \in l_{1}$. Then the vectors $y_{n}=x_{n}-y$ tend to $z=x_{0}-y$ with respect to $\tau$ and tend to 0 coordinatewise. We can also assume that the limits $\lim _{n \rightarrow \infty}\left\|y_{n}\right\|$ and $s=\lim _{n \rightarrow \infty} s\left(y_{n}\right)$ exist. Using Proposition 3, we see that

$$
\begin{aligned}
2 & =\lim _{n \rightarrow \infty}\left\|x_{0}-x_{n}\right\|=\lim _{n \rightarrow \infty}\left\|z-y_{n}\right\| \\
& =\|z\|+\lim _{n \rightarrow \infty}\left\|y_{n}\right\| \\
& \leqslant \lim _{m \rightarrow \infty}\left\|y_{m}\right\|+\lim _{n \rightarrow \infty}\left\|y_{n}\right\| \\
& =\lim _{m \rightarrow \infty} \lim _{n \rightarrow \infty}\left\|y_{m}-y_{n}\right\|=2 .
\end{aligned}
$$

This shows that $\lim _{n \rightarrow \infty}\left\|y_{n}\right\|=1=\|z\|$ and consequently, $|s| \leqslant 1$.

But $z=$ se. It follows that $\|e\|=1$ and $|s|=1$. We therefore see that (i) does not hold, so by our assumption the set $N^{+}$is finite. Consider the case when $s=1$. We choose $n$ for which $s\left(y_{n}\right)>1 / 2$ and $\left\|P_{m}\left(y_{n}\right)\right\|<1 / 4$ where $m=\max N^{+}$. Then the set $B=\left\{k \in \mathbb{N}: e(k) y_{n}(k)<0\right\}$ is nonempty. It is easy to see that

$$
|a+b|=|a|+|b|-2 \min \{|a|,|b|\}
$$

whenever $a, b \in \mathbb{R}, a b<0$. Consequently,

$$
\left\|e+y_{n}\right\|=\|e\|+\left\|y_{n}\right\|-2 c
$$

where $c=\sum_{k \in B} \min \left\{|e(k)|,\left|y_{n}(k)\right|\right\}>0$. Applying Proposition 3, we therefore obtain

$$
\begin{aligned}
\lim _{m \rightarrow \infty}\left\|\frac{1}{2}\left(x_{0}+x_{n}\right)-x_{m}\right\| & =\lim _{m \rightarrow \infty}\left\|\frac{1}{2}\left(z+y_{n}\right)-y_{m}\right\| \\
& =\frac{1}{2}\left\|z+y_{n}\right\|+\lim _{m \rightarrow \infty}\left\|y_{m}\right\| \\
& =\frac{1}{2}\left(\lim _{m \rightarrow \infty}\left\|z-y_{m}\right\|+\lim _{m \rightarrow \infty}\left\|y_{n}-y_{m}\right\|\right)-c \\
& =2-c<2
\end{aligned}
$$

which is a contradiction. The case when $s=-1$ is similar.

We have proved that if (i) or (ii) holds, then $l_{1}$ has the $\tau$-fixed point property and from Theorem 7 we know that if $\|e\|>1$, then $l_{1}$ does not have this property. To complete the proof it therefore remains to show that if $\|e\|=1$ and the set $N^{+}$is infinite, 
then $l_{1}$ lacks the $\tau$-fixed point property. Let $\left(n_{k}\right)$ be an infinite sequence in $N^{+}$such that $w_{0}=e-u_{0} \neq 0$ where $u_{0}=\sum_{k=1}^{\infty} e\left(n_{k}\right) e_{n_{k}}$. We set $w=\left(1 /\left\|w_{0}\right\|\right) w_{0}$ and

$$
C=\left\{\mu_{1} e+\mu_{2} w+\sum_{k=1}^{\infty} \mu_{k+2} e_{n_{k}}: \sum_{j=1}^{\infty} \mu_{j}=1, \mu_{j} \geqslant 0, j=1,2, \ldots\right\} .
$$

The set $C$ is bounded and convex. By Corollary 2 it is also $\tau$-sequentially compact.

Moreover, if $\sum_{j=1}^{\infty} \mu_{j}=1$ and $\mu_{j} \geqslant 0$ for all $j \in \mathbb{N}$ then

$$
\mu_{1} e+\mu_{2} w+\sum_{k=1}^{\infty} \mu_{k+2} e_{n_{k}}=\left(\mu_{1}\left\|w_{0}\right\|+\mu_{2}\right) w+\sum_{k=1}^{\infty}\left(\mu_{1} e\left(n_{k}\right)+\mu_{k+2}\right) e_{n_{k}}
$$

and

$$
\mu_{1}\left\|w_{0}\right\|+\mu_{2}+\sum_{k=1}^{\infty}\left(\mu_{1} e\left(n_{k}\right)+\mu_{k+2}\right)=\mu_{1}\left(\left\|w_{0}\right\|+\left\|u_{0}\right\|\right)+\sum_{k=1}^{\infty} \mu_{k+1}=1
$$

It follows that

$$
C=\left\{\lambda_{0} w+\sum_{k=1}^{\infty} \lambda_{k} e_{n_{k}}: \sum_{j=0}^{\infty} \lambda_{j}=1, \lambda_{j} \geqslant 0, j=0,1,2, \ldots\right\} .
$$

Now, given $x=\lambda_{0} w+\sum_{k=1}^{\infty} \lambda_{k} e_{n_{k}} \in C$, we put

$$
T(x)=\sum_{k=0}^{\infty} \lambda_{k} e_{n_{k+1}}
$$

It is easy to see that $T: C \rightarrow C$ is an isometry and does not have a fixed point in $C$.

The reasoning in the first part of the proof can be also used to show that if $\|e\|<1$, then $l_{1}$ has the Kadec-Klee $(\tau)$ property. By Corollary 6 , in this case $l_{1}$ has the fixed point property for mappings of asymptotically nonexpansive type on bounded convex $\tau$-sequentially compact sets. The standard example of such topology is $\tau=\sigma\left(l_{1}, c_{0}\right)$. The sequence $\left(e_{n}\right)$ converges to 0 with respect to this topology. Observe in turn that if $\tau=\sigma\left(l_{1}, c\right)$, then $\left(e_{n}\right)$ converges to the vector $(1,0,0, \ldots)$. Changing the basis of $l_{1}$, we can generalise the last example. Namely, given $e \in l_{1}, e \neq 0$, we set $m=\min$ suppe, $e_{m}^{\prime}=e$ and $e_{k}^{\prime}=e_{k}$ if $k \neq m$. Then the sequence $\left(e_{n}^{\prime}\right)$ is a basis of $l_{1}$ equivalent to $\left(e_{n}\right)$. It follows that the subspace

$$
Y=\left\{x^{*} \in l_{1}^{*}: x^{*}(e)=\lim _{n \rightarrow \infty} x^{*}\left(e_{n}\right)\right\}
$$

is total. Consequently, $\tau=\sigma\left(l_{1}, Y\right)$ is a Hausdorff topology and clearly $e$ is the $\tau$-limit of $\left(e_{n}\right)$. 
Let now $\Gamma$ be an arbitrary infinite set. By $\left(e_{i}\right)_{i \in \Gamma}$ we denote the standard basis of the real space $l_{1}(\Gamma)$. Assume that $\tau$ is a locally convex topology in $X=l_{1}(\Gamma)$ such that $\tau_{B_{X}}$ is coarser than $w_{B_{X}}$. The reasoning from the last part of the proof of Theorem 8 can be used to show that if some sequence $\left(e_{i_{n}}\right)$ converges with respect to $\tau$ to a norm-one element $e \in l_{1}(\Gamma)$ and the set $N^{+}=\left\{n \in \mathbb{N}: e\left(i_{n}\right) \geqslant 0\right\}$ is infinite, then $l_{1}(\Gamma)$ does not have the $\tau$-fixed point property. Actually we obtain a fixed point free isometry, but using the reasoning from the proof of Theorem 7, we can also get a fixed point free contractive mapping.

As an example consider a scattered compact topological space $\Gamma$ (see [6, p. 398]). Then $C(\Gamma)^{*}$ can be identified with $l_{1}(\Gamma)$ with the duality formula

$$
x(f)=\sum_{i \in \operatorname{supp} x} x(i) f(i)
$$

where $x \in l_{1}(\Gamma)$ is not zero and $f \in C(\Gamma)$. The space $\Gamma$ is sequentially compact (see [6, p. 419]), so if it is infinite, then there exists a sequence $\left(\gamma_{n}\right)$ of distinct points of $\Gamma$ converging to some $\gamma \in \Gamma$. Clearly, $\left(e_{\gamma_{n}}\right)$ converges to $e_{\gamma}$ with respect to the weak* topology $\tau=\sigma\left(l_{1}(\Gamma), C(\Gamma)\right)$. We therefore see that $l_{1}(\Gamma)$ does not have the $\tau$-fixed point property.

\section{4. $c_{0}$-SUMS OF REFLEXIVE SPACES}

In $[4,5]$, it was proved that if a closed bounded convex subset $C$ of $c_{0}$ is not weakly compact, then there is a nonexpansive mapping $T: C \rightarrow C$ without a fixed point. We shall use an idea from the first of these papers to obtain the following result.

THEOREM 9. Let $\left(X_{n}\right)$ be a sequence of reflexive Banach spaces and $X=\left(\sum_{i=1}^{\infty} X_{i}\right)_{c_{0}}$. If $\tau$ is a locally convex topology in $X$ such that $\tau_{B_{X}}$ is strictly coarser than $w_{B_{X}}$, then there exist a bounded convex $\tau$-sequentially compact set $C \subset X$ and a contractive mapping $T: C \rightarrow C$ which does not have a fixed point.

Proof: Let $X^{\prime}$ be the space of all $x^{*} \in X^{*}$ such that $x_{\mid B_{X}}^{*}$ is $\tau_{B_{X}}$-continuous. It is easy to see that $X^{\prime}$ is a norm closed subspace of $X^{*}$ and since $\tau_{B_{X}}$ is strictly coarser than $w_{B_{X}}, X^{\prime} \neq X^{*}$. We can therefore find $z^{* *} \in X^{* *}$ such that $z^{* *} \neq 0$ and $z^{* *}(f)=0$ for every $f \in X^{\prime}$. Since $\tau$ is a Hausdorff topology, the subspace $X^{\prime}$ is total. Consequently, $z^{* *} \notin X$. Notice that

$$
X^{* *}=\left(\sum_{i=1}^{\infty} X_{i}\right)_{l_{\infty}}
$$

so $z^{* *}=(z(i))$ where $z_{i} \in X_{i}$ for every $i \in \mathbb{N}$ and $\left\|z^{* *}\right\|=\sup _{i \in \mathbb{N}}\|z(i)\|_{X_{i}}<+\infty$.

Given $n \in \mathbb{N}$ and $v^{* *}=(v(i)) \in X^{* *}$, we put $P_{n}\left(v^{* *}\right)=(v(1), \ldots, v(n), 0,0, \ldots)$ and $R_{n}\left(v^{* *}\right)=v^{* *}-P_{n}\left(v^{* *}\right)$. In addition, we set $R_{0}\left(v^{* *}\right)=v^{* *}$ and $n_{0}=0$. We find $n_{1} \in \mathbb{N}$ 
so that $(4 / 5)\left\|z^{* *}\right\| \leqslant\left\|P_{n_{1}}\left(z^{* *}\right)\right\|$. Next, having $n_{1}<\cdots<n_{k}$, we choose $n_{k+1}>n_{k}$ such that

$$
\frac{4^{k+1}}{1+4^{k+1}}\left\|R_{n_{k}}\left(z^{* *}\right)\right\|<\left\|P_{n_{k+1}}\left(R_{n_{k}}\left(z^{* *}\right)\right)\right\|
$$

This inductive procedure gives us an increasing sequence $\left(n_{k}\right)$. We set $x_{1}=P_{n_{1}}\left(z^{* *}\right)$ and $x_{k}=P_{n_{k}}\left(R_{n_{k-1}}\left(z^{* *}\right)\right)$ if $k>1$. Then

$$
\left\|x_{i}\right\| \leqslant\left\|R_{n_{k-1}}\left(z^{* *}\right)\right\| \leqslant\left(1+\frac{1}{4^{k}}\right)\left\|x_{k}\right\|
$$

for every $i>k$.

We also put $w_{k}=P_{n_{k}}\left(z^{* *}\right)=\sum_{i=1}^{k} x_{i}$. It is easy to see that

$$
\left\|\sum_{k=1}^{\infty} \alpha_{k} w_{k}\right\|=\sup _{i \in \mathbb{N}}\left|\sum_{j=i}^{\infty} \alpha_{j}\right|\left\|x_{i}\right\|
$$

for each sequence of scalars $\left(\alpha_{k}\right)$ such that the series $\sum_{n=1}^{\infty} \alpha_{n}$ converges. This shows that $\left(w_{n}\right)$ is a basic sequence in $X$ equivalent to the summing basis of $c_{0}$.

Consider now the following subset of $X$

$$
C=\left\{\sum_{n=1}^{\infty} t_{n} w_{n}: \sum_{n=1}^{\infty} t_{n} \leqslant 1, t_{n} \geqslant 0, n=1,2, \ldots\right\} .
$$

It is clear that $C$ is convex and bounded. In order to prove that $C$ is $\tau$-sequentially compact observe that $\left(w_{n}\right)$ converges to $z^{* *}$ with respect to the topology $\sigma\left(X^{* *}, X^{*}\right)$. If $f$ is a linear functional on $X$ continuous with respect to $\tau$, then $f \in X^{\prime}$ and consequently, $\lim _{n \rightarrow \infty} f\left(w_{n}\right)=z^{* *}(f)=0$. We therefore see that $\left(w_{n}\right)$ converges to 0 with respect to $\tau$. This and Corollary 2 show that $C$ is $\tau$-sequentially compact.

To construct a fixed point free nonexpansive mapping $T: C \rightarrow C$ we follow an idea from [4]. Namely, we put

$$
u_{n}=\sum_{j=1}^{\infty} \frac{1}{2^{j}} w_{n+j}
$$

for $n=0,1, \ldots$ Next, given $u=\sum_{k=1}^{\infty} t_{k} w_{k} \in C$, we set

$$
T(u)=\left(1-\sum_{n=1}^{\infty} t_{n}\right) u_{0}+\sum_{n=1}^{\infty} t_{n} u_{n}
$$

It is easy to see that

$$
T(u)=\frac{1}{2}\left(1-\sum_{n=1}^{\infty} t_{n}\right) w_{1}+\sum_{k=2}^{\infty}\left(\frac{1}{2^{k}}\left(1-\sum_{n=1}^{\infty} t_{n}\right)+\sum_{i+j=k} \frac{1}{2^{i}} t_{j}\right) w_{k}
$$


This shows that $T$ is well defined. Moreover, if $T(u)=u$, then $\left(1-\sum_{n=1}^{\infty} t_{n}\right) / 2=t_{1}$, so $t_{2}=\left(1-\sum_{n=1}^{\infty} t_{n}\right) / 4+t_{1} / 2=t_{1}$ and similarly, $t_{k}=t_{1}$ for every $k$. Thus $t_{k}=0$ for all $k$, which means that $u=0$. But $T(0)=u_{0} \neq 0$. This contradiction shows that $T$ does not have a fixed point.

It remains to prove that $T$ is nonexpansive. We fix elements $x=\sum_{n=1}^{\infty} t_{n} w_{n}$, $y=\sum_{n=1}^{\infty} s_{n} w_{n}$ in $C$ and set $\alpha_{0}=\sum_{n=1}^{\infty}\left(s_{n}-t_{n}\right)$ and $\alpha_{k}=t_{k}-s_{k}$ for $k \geqslant 1$. Then $\sum_{n=0}^{\infty} \alpha_{n}=0$. Easy calculations give

$$
\left\|P_{n_{1}}(T(x)-T(y))\right\|=\left\|x_{1}\right\|\left|\sum_{n=0}^{\infty} \alpha_{n}\right|=0
$$

and

$$
\begin{aligned}
\left\|P_{n_{m}}\left(R_{n_{m-1}}(T(x)-T(y))\right)\right\| & =\left\|x_{m}\right\|\left|\sum_{k=m}^{\infty}\left(\frac{1}{2^{k}} \alpha_{0}+\sum_{i+j=k} \frac{1}{2^{i}} \alpha_{j}\right)\right| \\
& =\left\|x_{m}\right\|\left|\left(1-\frac{1}{2^{m-1}}\right) \sum_{k=1}^{\infty} \alpha_{k}-\sum_{i=1}^{m-2}\left(1-\frac{1}{2^{m-1-i}}\right) \alpha_{i}\right| \\
& =\left\|x_{m}\right\|\left|\sum_{k=1}^{m-1} \frac{1}{2^{m-k}} \sum_{i=k}^{\infty} \alpha_{i}\right|
\end{aligned}
$$

for every $m>1$. This, (2), and (3) show that

$$
\begin{aligned}
\left\|P_{n_{m}}\left(R_{n_{m-1}}(T(x)-T(y))\right)\right\| & \leqslant \sum_{k=1}^{m-1} \frac{1}{2^{m-k}}\left(1+\frac{1}{4^{k}}\right)\left\|x_{k}\right\|\left|\sum_{i=k}^{\infty} \alpha_{i}\right| . \\
& \leqslant\|x-y\|
\end{aligned}
$$

and the first inequality becomes strict if $\alpha_{k} \neq 0$ for some $1 \leqslant k \leqslant m-2$. Hence

$$
\|T(x)-T(y)\|=\max _{m>1}\left\|P_{n_{m}}\left(R_{n_{m-1}}(T(x)-T(y))\right)\right\|<\|x-y\|
$$

for all $x, y \in C$ with $x \neq y$.

Let $X$ be a Banach space. The geometric coefficient $R(X)$ is defined as

$$
R(X)=\sup \left\{\liminf _{n \rightarrow \infty}\left\|x_{n}+x\right\|\right\}
$$

where the supremum is taken over all $x \in B_{X}$ and all weakly null sequences $\left(x_{n}\right)$ in $B_{X}$. This coefficient is related to existence of fixed points of nonexpansive mappings in the following way (see [7]): 
Theorem 10. Let $X$ be a Banach space. If $R(X)<2$, then $X$ has the $w$-fixed point property.

Using $R(X)$ we can give a positive result concerning fixed point property for nonexpansive mappings for $c_{0}$-sums of Banach spaces.

TheOREm 11. Let $\Gamma$ be an infinite set, $\left(X_{i}\right)_{i \in \Gamma}$ be a collection of Banach spaces and $X=\left(\sum_{i \in \Gamma} X_{i}\right)_{c_{0}(\Gamma)}$. Then $R(X)=\sup _{i \in \Gamma} R\left(X_{i}\right)$.

Proof: It is clear that $R(X) \geqslant R\left(X_{i}\right)$ for every $i \in \Gamma$. So we only need to prove that $R(X) \leqslant \sup _{i \in \Gamma} R\left(X_{i}\right)$. We fix $\varepsilon>0, x \in B_{X}$ and a weakly null sequence $\left(x_{n}\right)$ in $B_{X}$. Thus $x_{n}=\left(x_{n}(i)\right)_{i \in \Gamma}$ and $x=(x(i))_{i \in \Gamma}$ where $x_{n}(i)$ and $x(i)$ belong to $X_{i}$ for every $i \in \Gamma$. Since $x \in X$, there exists some finite subset $F \subset \Gamma$ such that $\|x(i)\|_{X_{i}} \leqslant \varepsilon$ if $i \in \Gamma \backslash F$. Clearly, $\sup _{i \in \Gamma \backslash F}\left\|x_{n}(i)+x(i)\right\|_{X_{i}} \leqslant 1+\varepsilon \leqslant \sup _{i \in \Gamma} R\left(X_{i}\right)+\varepsilon$ for every $n \in \mathbb{N}$.

We can assume that the limits $\lim _{n \rightarrow \infty}\left\|x_{n}+x\right\|$ and $\lim _{n \rightarrow \infty}\left\|x_{n}(i)+x(i)\right\|_{X_{i}}$ exist for every $i \in F$. Then we can find $n_{0} \in \mathbb{N}$ such that

$$
\left\|x_{n}(i)+x(i)\right\|_{X_{i}} \leqslant \lim _{n \rightarrow \infty}\left\|x_{n}(i)+x(i)\right\|_{X_{i}}+\varepsilon \leqslant R\left(X_{i}\right)+\varepsilon \leqslant \sup _{i \in \Gamma} R\left(X_{i}\right)+\varepsilon
$$

for every $n \geqslant n_{0}$ and for every $i \in F$. Thus $\sup _{i \in F}\left\|x_{n}(i)+x(i)\right\|_{X_{i}} \leqslant \sup _{i \in \Gamma} R\left(X_{i}\right)+\varepsilon$ for every $n \geqslant n_{0}$. Passing to limits when $n$ goes to infinity, we obtain

$$
\lim _{n \rightarrow \infty}\left\|x_{n}+x\right\|=\lim _{n \rightarrow \infty} \sup _{i \in \Gamma}\left\|x_{n}(i)+x(i)\right\|_{X_{i}} \leqslant \sup _{i \in \Gamma} R\left(X_{i}\right)+\varepsilon,
$$

and since $\varepsilon>0$ is arbitrary, we get the desired inequality.

COROLLARY 12. Let $\left(X_{n}\right)$ a sequence of reflexive Banach spaces such that $\sup _{i \in \mathbb{N}} R\left(X_{i}\right)<2$. Then $X=\left(\sum_{n=1}^{\infty} X_{i}\right)_{c_{0}}$ has the $w$-fixed point property and fails to have the $\tau$-fixed point property for every locally convex topology $\tau$ in $X$ such that $\tau_{B_{X}}$ is strictly coarser than $w_{B_{X}}$.

We have $R\left(l_{p}\right)=2^{1 / p}$ for every $1<p<+\infty$ (see [7]). Let $\left(p_{n}\right)$ be a sequence in $(1,+\infty)$ such that $\inf _{n \in \mathbb{N}} p_{n}>1$ and $X=\left(\sum_{n=1}^{\infty} l_{p_{n}}\right)_{c_{0}}$. Then from Corollary 12 we see that $X$ has the $w$-fixed point property and fails to have the $\tau$-fixed point property for every locally convex topology $\tau$ in $X$ such that $\tau_{B_{X}}$ is strictly coarser than $w_{B_{X}}$.

\section{REFERENCES}

[1] T.D. Benavides and P. Lorenzo Ramirez, 'Structure of the fixed point set and common fixed points of asymptotically nonexpansive mappings', Proc. Amer. Math. Soc. 129 (2001), 3549-3557. 
[2] T.D. Benavides, J. García Falset and M.A. Japón Pineda, 'The $\tau$-fixed point property for nonexpansive mappings', Abstract Appl. Anal. 3 (1998), 343-362.

[3] T.D. Benavides, M.A. Japón Pineda and S. Prus, 'Weak compactness and fixed point property for affine mappings', J. Functional Anal. 209 (2004), 1-15.

[4] P.N. Dowling. C.J. Lennard and B. Turett, 'Characterizations of weakly compact sets and new fixed point free maps in $c_{0}$ ', Studia Math. 154 (2003), 277-293.

[5] P.N. Dowling. C.J. Lennard and B. Turett, 'Weakly compactness is equivalent to the fixed point property in $c_{0}$ ', Proc. Amer. Math. Soc. 132 (2004), 1659-1666.

[6] M. Fabian, P. Habala, P. Hájek, V. Montesinos Santalucía, J. Pelant and V. Zizler, Functional analysis and infinite-dimensional geometry (Springer-Verlag, New York, 2001).

[7] J. García Falset, 'The fixed point property in Banach spaces with NUS-property', $J$. Math. Anal. Appl. 215 (1997), 532-542.

[8] K. Goebel and T. Kuczumow, 'Irregular convex sets with fixed point property for nonexpansive mappings', Colloq. Math. 40 (1979), 259-264.

[9] P. Harmand, D. Werner and W. Werner, $M$-ideals in Banach spaces and Banach algebras (Springer-Verlag, Berlin, 1993).

[10] M.A. Japón Pineda, Stability of the fixed point property for nonexpansive mappings, (Ph. D. Dissertation) (Seville, 1998).

[11] M.A. Japón Pineda, 'Existence of fixed points for mappings of asymptotically nonexpansive type on L-embedded Banach spaces', Nonlinear Anal. 47 (2001), 2779-2786.

[12] M.A. Japón Pineda, 'Some fixed points results on $L$-embedded Banach spaces', $J$. Math. Anal. Appl. 272 (2002), 380-391.

[13] L.V. Kantorovich and G.P. Akilov, Functional analysis (Pergamon Press, OxfordElmsford, New York, 1982).

[14] C.J. Lennard, 'A new convexity property that implies a fixed property for $L_{1}$ ', Studia Math. 100 (1991), 95-108.

[15] M. Nowak, 'Compact Hankel operators with conjugate analytic symbols', Rend. Circ. Mat. Palermo (2) 47 (1998), 363-374.

[16] H. Pfitzner, ' $L$-embedded Banach spaces and convergence in measure', (preprint).

[17] J.L. Ramírez, Some deterministic and random metric fixed point theorems, (Ph. D. Dissertation) (Seville, 2002).

[18] H.K. Xu, 'Existence and convergence for fixed points of mappings of asymptotically nonexpansive type', Nonlinear Anal. 16 (1991), 1139-1146.

Departamento de Análisis Matemático

Universidad de Sevilla

41080 Sevilla

Spain

e-mail: japon@us.es
Institute of Mathematics

Maria Curie-Skłodowska University

20-031 Lublin

Poland

e-mail: bsprus@golem.umcs.lublin.pl 